\title{
Order preserving SUPG stabilization for the Virtual Element formulation of advection-diffusion problems ${ }^{\text {th }}$
}

\author{
M. F. Benedetto, S. Berrone*, A. Borio, S. Pieraccini, S. Scialò \\ Dipartimento di Scienze Matematiche, Politecnico di Torino \\ Corso Duca degli Abruzzi 24, Torino, 10129, Italy
}

\begin{abstract}
In the framework of the discretization of advection-diffusion problems by means of the Virtual Element Method, we consider stabilization issues. Herein, stabilization is pursued by adding a consistent SUPG-like term. For this approach we prove optimal rates of convergence. Numerical results clearly show the stabilizing effect of the method up to very large Péclet numbers and are in very good agreement with the expected rate of convergence.
\end{abstract}

Keywords: Virtual Element Methods, Advection-diffusion problem, SUPG, stability, convergence

2000 MSC: 65N30, 65M12

\section{Introduction}

Recently, a new discretization approach has been developed, named the Virtual Element Method (VEM), that allows the use of general polygonal and polyhedral meshes [1, 2].

The VEM has been applied in a wide number of contexts, such as plate bending problems [3], elasticity problems [4, 5, Stokes problems [6] and the Steklov eigenvalue problem [7]. A non-conforming formulation has been devised in [8]. Recently, the VEM has been also used in the treatment of fluid dynamics models involving underground flow simulations [11: in that context, the application of the VEM was driven by the need of circumventing mesh generation problems. In these applications, the primal problem is solved to compute the Darcy velocity field, that can be used afterwards to simulate the transport of a dispersed, passive pollutant in a geological basin. The flow regimes in underground transport phenomena are usually transport-dominated, due to the very low diffusivity of the pollutant into the bulk fluid, thus calling for a stabilization of the VEM.

This research has been partially supported by the Italian MIUR through PRIN research grant 2012HBLYE4_001 Metodologie innovative nella modellistica differenziale numerica and by INdAMGNCS. Matías Fernando Benedetto was supported by the European Commission through the Erasmus Mundus Action 2-Strand1 ARCOIRIS programme, Politecnico di Torino.

${ }^{*}$ Corresponding author

Preprint submitted to Elsevier

April 14, 2016 
Many strategies have been devised to obtain a stable solution for standard Finite Element discretizations, involving, for example, local projections 12 or suitably built bubble functions [13, 14. The Streamline Upwind Petrov-Galerkin (SUPG) stabilization method [15 21] has also been widely studied in very general settings. A first approach to the VEM-SUPG stabilization is discussed in [22], in a non-consistent formulation.

Another issue related to advection-diffusion problems is the derivation of robust a posteriori error estimates. In such context, the term robustness refers to the property of obtaining a relation between the error and the error estimator with constants which are independent of the Péclet number 23 28. An a posteriori analysis for the reactionconvection-diffusion problem with the VEM is provided in [29, not addressing robustness aspects and the SUPG-like stabilization issues.

The aim of this work is to devise a consistent SUPG formulation compatible with the VEM. A key aspect of the VEM is that the basis functions of the discrete functional space are not known explicitly, but only through their degrees of freedom. As a consequence, computability of discrete operators requires special care and, in particular, the consistent VEM-SUPG formulation devised in the present work requires the introduction of a second-order term in the weak formulation of the problem, computed by resorting to polynomial projections of the virtual element basis functions.

An a priori error estimate for the stabilized VEM discrete solution is also proven, showing that the order of convergence is not affected by the stabilizing perturbation added to the problem. Numerical tests proposed in the paper confirm the theoretical results on triangular and polygonal meshes in both the convection-dominated regime and the diffusion-dominated regime.

The paper is organised as follows. In Section 2 we state the model problem, define some useful notations and make some standard hypothesis on the model parameters. In Section 3 we introduce the spatial discretization and the Virtual Element functional space based on it. In particular, the VEM-SUPG formulation of the problem is presented in Subsection 3.1, equations (13), 19 and 20p. In Section 4 the a priori error estimate for the stabilized VEM discrete solution is derived, the main result being stated in Theorem 2. Finally, in Section 5 we propose some numerical tests aimed at confirming the theoretical results.

\section{The model problem}

Let $\Omega \subset \mathbb{R}^{2}$ be a bounded open set and let us consider the following convectiondiffusion problem:

$$
\begin{cases}-\nabla \cdot(\mathrm{K} \nabla u)+\beta \cdot \nabla u=f & \text { in } \Omega, \\ u=0 & \text { on } \partial \Omega,\end{cases}
$$

where $\mathrm{K} \in \mathrm{L}^{\infty}(\Omega)$ is a positive function satisfying $\mathrm{K}(x) \geq \mathrm{K}_{0} \forall x \in \Omega$ for a given $\mathrm{K}_{0}>0$, and $\beta \in\left[\mathrm{L}^{\infty}(\Omega)\right]^{2}, \nabla \cdot \beta \in \mathrm{L}^{2}(\Omega)$. We additionally assume $\nabla \cdot \beta=0$.

The notation throughout the paper is as follows: $(\cdot, \cdot)$ and $\|\cdot\|$ denote the $\mathrm{L}^{2}(\Omega)$ scalar products and norms; $(\cdot, \cdot)_{\omega}$ and $\|\cdot\|_{\omega}$ denote the $\mathrm{L}^{2}(\omega)$ scalar products and norms, for any $\omega \subseteq \Omega$; moreover, $\|\cdot\|_{\alpha}$ and $|\cdot|_{\alpha}$ denote the $\mathrm{H}^{\alpha}(\Omega)$ norm and semi-norm; $\|\cdot\|_{\alpha, \omega}$ and $|\cdot|_{\alpha, \omega}$ denote the $\mathrm{H}^{\alpha}(\omega)$ norm and semi-norm; whereas $\|\cdot\|_{\mathrm{W}_{p}^{q}(\omega)}$ and $|\cdot|_{\mathrm{W}_{p}^{q}(\omega)}$ denote the $\mathrm{W}_{p}^{q}(\omega)$ norm and semi-norm, where $p$ is the Lebesgue regularity and $q$ is the order of the Sobolev space. 
For future reference, we recall the classical weak formulation of the problem. Defining $B: \mathrm{H}_{0}^{1}(\Omega) \times \mathrm{H}_{0}^{1}(\Omega) \rightarrow \mathbb{R}$ and $F: \mathrm{H}_{0}^{1}(\Omega) \rightarrow \mathbb{R}$ such that

$$
B(w, v):=(\mathrm{K} \nabla w, \nabla v)+(\beta \cdot \nabla w, v) \quad \forall w, v \in \mathrm{H}_{0}^{1}(\Omega),
$$

and

$$
F(v):=(f, v) \quad \forall v \in \mathrm{H}_{0}^{1}(\Omega),
$$

the variational form of (1) is

$$
B(u, v)=F(v) \quad \forall v \in \mathrm{H}_{0}^{1}(\Omega) .
$$

We remark that, for the sake of improving readability, here we limit ourself to formulation (2). More general boundary conditions can be considered as well. Furthermore, K can be taken as a symmetric positive definite tensor, with minor changes in some definitions.

\section{VEM discretization}

Let $\mathcal{T}_{h}$ be a set of open polygons partitioning $\Omega, h$ being the maximum diameter of these elements. For VEM-based discretizations these polygons can have a different number of edges from one to another and also nodes can be placed between edges forming a flat angle, thus allowing for hanging-node-like configurations. As usually done for VEM discretizations (see [2]), we ask that every polygon is star-shaped with respect to a ball whose radius is greater or equal than $\gamma h_{E}$, being $h_{E}$ the element diameter and $\gamma$ a global constant. Finally, for each $E \in \mathcal{T}_{h}$, we set

$$
\begin{array}{rlrl}
\mathrm{K}_{E} & :=\sup _{x \in E} \mathrm{~K}(x), & \mathrm{K}_{E}^{\vee}:=\inf _{x \in E} \mathrm{~K}(x), \\
\beta_{E}:=\sup _{x \in E}\|\beta(x)\|_{\mathbb{R}^{2}}^{2} . &
\end{array}
$$

To define the Virtual Element space of order $k>0$, for some $k \in \mathbb{N}$, we denote by $\mathbb{P}_{k}\left(\mathcal{T}_{h}\right)$ the space of possibly discontinuous functions which are polynomials of degree less than or equal to $k$ on each polygon and we introduce the piecewise polynomial oblique projection $\Pi_{k}^{\nabla}: \mathrm{H}^{1}(E) \rightarrow \mathbb{P}_{k}\left(\mathcal{T}_{h}\right)$ such that, $\forall E \in \mathcal{T}_{h}$,

$$
\begin{aligned}
& \left(\nabla\left(v-\Pi_{k}^{\nabla} v\right), \nabla p\right)_{E}=0 \quad \forall p \in \mathbb{P}_{k}(E), \\
& \quad \text { and } \quad\left(\Pi_{k, E}^{\nabla} v, 1\right)_{\partial E}=(v, 1)_{\partial E} .
\end{aligned}
$$

The local Virtual space of order $k$ is defined as follows: $\forall E \in \mathcal{T}_{h}$,

$$
\begin{aligned}
& V_{h}^{E}:=\left\{v_{h} \in \mathrm{H}^{1}(E): \Delta v_{h} \in \mathbb{P}_{k}(E), v_{h} \in \mathbb{P}_{k}(e) \forall e \subset \partial E\right. \\
&\left.\left(v_{h}, p\right)_{E}=\left(\Pi_{k, E}^{\nabla} v_{h}, p\right)_{E} \forall p \in \mathbb{P}_{k}(E) \backslash \mathbb{P}_{k-2}(E)\right\},
\end{aligned}
$$

and, asking for global continuity we obtain the global space $V_{h} \subset \mathrm{H}_{0}^{1}(\Omega)$ :

$$
V_{h}:=\left\{v_{h} \in C^{0}(\Omega): v_{h} \in V_{h}^{E} \quad \forall E \in \mathcal{T}_{h}\right\} .
$$

A function belonging to such space is uniquely identified by its polynomial expression on each edge of the discretization and by its moments against polynomials of degree $\leq k-2$ (see [1]). As in [1, we choose the following set of degrees of freedom (see also [30] for more details): 
1. the values at the vertices of each polygon;

2. if $k \geq 2$, for each edge $e \subset \partial E$, the values at $k-1$ internal points of $e$. For practical purposes, we may choose these points to be the internal Gauss-Lobatto quadrature nodes;

3. if $k \geq 2$, for each $v_{h} \in V_{h}$ the moments $\left(v_{h}, m_{\boldsymbol{\alpha}}\right)_{E}$, for each $E \in \mathcal{T}_{h}$ and all the monomials $m_{\boldsymbol{\alpha}} \in \mathcal{M}_{k-2}(E)$, defined as

$$
m_{\boldsymbol{\alpha}}(x, y):=\frac{\left(x-x_{E}\right)^{\alpha_{1}}\left(y-y_{E}\right)^{\alpha_{2}}}{h_{E}^{\alpha_{1}+\alpha_{2}}} \quad \forall(x, y) \in E,
$$

with $\boldsymbol{\alpha}=\left(\alpha_{1}, \alpha_{2}\right),|\boldsymbol{\alpha}|=\alpha_{1}+\alpha_{2} \leq k-2$.

\subsection{VEM-SUPG formulation}

It is well known that discretizing the variational formulation (2) leads to instabilities when the convective term $(\beta \cdot \nabla w, v)$ is dominant with respect to the diffusive term $(\mathrm{K} \nabla w, \nabla v)$. In such situations a stabilized form of the problem is required in order to prevent spurious oscillations that can completely alter the numerical solution. In the following we recast the classical Streamline Upwind Petrov Galerkin (SUPG) approach [17. in the framework of the VEM, showing that the optimal order of convergence can be preserved.

We define the bilinear form $B_{\text {supg }}: \mathrm{H}^{2}(\Omega) \cap \mathrm{H}_{0}^{1}(\Omega) \times \mathrm{H}_{0}^{1}(\Omega) \rightarrow \mathbb{R}$ such that

$$
B_{\text {supg }}(w, v):=a(w, v)+b(w, v)+d(w, v),
$$

being

$$
\begin{aligned}
a(w, v) & :=(\mathrm{K} \nabla w, \nabla v)+\sum_{E \in \mathcal{T}_{h}} \tau_{E}(\beta \cdot \nabla w, \beta \cdot \nabla v), \\
b(w, v) & :=(\beta \cdot \nabla w, v), \\
d(w, v) & :=-\sum_{E \in \mathcal{T}_{h}} \tau_{E}(\nabla \cdot(\mathrm{K} \nabla w), \beta \cdot \nabla v)_{E} .
\end{aligned}
$$

The stability parameter $\tau_{E}$ is defined as usual, $\forall E \in \mathcal{T}_{h}$, by

$$
\tau_{E}:=\frac{h_{E}}{2 \beta_{E}} \min \left\{\mathrm{Pe}_{E}, 1\right\},
$$

where $\mathrm{Pe}_{E}$ is the mesh Péclet number of $E$, given by

$$
\mathrm{Pe}_{E}:=m_{k}^{E} \frac{\beta_{E} h_{E}}{2 \mathrm{~K}_{E}},
$$

and

$$
m_{k}^{E}:= \begin{cases}\frac{1}{3} & \text { if } \nabla \cdot\left(\mathrm{K} \nabla v_{h}\right)=0 \forall v_{h} \in V_{h}^{E}, \\ 2 \tilde{C}_{k}^{E} & \text { otherwhise }\end{cases}
$$

having set $\tilde{C}_{k}^{E}$ to be the largest constant satisfying the following inverse inequality:

$$
\tilde{C}_{k}^{E} h_{E}^{2}\left\|\nabla \cdot\left(\mathrm{K} \nabla v_{h}\right)\right\|_{E}^{2} \leq\left\|\mathrm{K} \nabla v_{h}\right\|_{E}^{2} \quad \forall v_{h} \in V_{h}^{E} .
$$


Remark 1 . We point out that if $u \in \mathrm{H}^{2}(\Omega)$, we have that

$$
B_{\text {supg }}(u, v)=F_{\text {supg }}(v):=(f, v)+\sum_{E \in \mathcal{T}_{h}} \tau_{E}(f, \beta \cdot \nabla v) \quad \forall v \in \mathrm{H}_{0}^{1}(\Omega) .
$$

Remark 2. From the definition of $\tau_{E}$ we have the following two estimates, that will be used in the following:

$$
\begin{gathered}
\tau_{E} \leq \frac{\tilde{C}_{k}^{E} h_{E}^{2}}{2 \mathrm{~K}_{E}} \quad \text { if } \nabla \cdot\left(\mathrm{K} \nabla v_{h}\right) \neq 0 \text { for some } v_{h} \in V_{h}^{E}, \\
\tau_{E} \leq \frac{h_{E}}{2 \beta_{E}} .
\end{gathered}
$$

The Finite Element discretization of the bilinear form (3) has been widely studied, for example in [15, 17, in which optimal orders of convergence were proved. In order to write a computable VEM discretization of problem (2), we define the discrete bilinear form $B_{\text {supg }, h}: V_{h} \times V_{h} \rightarrow \mathbb{R}$ such that

$$
B_{\text {supg }, h}\left(w_{h}, v_{h}\right):=a_{h}\left(w_{h}, v_{h}\right)+b_{h}\left(w_{h}, v_{h}\right)+d_{h}\left(w_{h}, v_{h}\right) \quad \forall w_{h}, v_{h} \in V_{h},
$$

where

$$
\begin{aligned}
a_{h}\left(w_{h}, v_{h}\right):= & \left(\mathrm{K} \Pi_{k-1}^{0} \nabla w_{h}, \Pi_{k-1}^{0} \nabla v_{h}\right) \\
& +\sum_{E \in \mathcal{T}_{h}} \tau_{E}\left(\beta \cdot \Pi_{k-1}^{0} \nabla w_{h}, \beta \cdot \Pi_{k-1}^{0} \nabla v_{h}\right)_{E} \\
& +\left(\mathrm{K}_{E}+\tau_{E} \beta_{E}^{2}\right) S^{E}\left(\left(I-\Pi_{k}^{\nabla}\right) w_{h},\left(I-\Pi_{k}^{\nabla}\right) v_{h}\right), \\
b_{h}\left(w_{h}, v_{h}\right):= & \left(\beta \cdot \Pi_{k-1}^{0} \nabla w_{h}, \Pi_{k-1}^{0} v\right), \\
d_{h}\left(w_{h}, v_{h}\right):= & -\sum_{E \in \mathcal{T}_{h}} \tau_{E}\left(\nabla \cdot\left(\mathrm{K} \Pi_{k-1}^{0} \nabla w_{h}\right), \beta \cdot \Pi_{k-1}^{0} \nabla v_{h}\right),
\end{aligned}
$$

where $\Pi_{r}^{0}$ is the element-wise orthogonal $\mathrm{L}^{2}$ projection on the space of polynomials of degree less than or equal to $r$, as used in [2]. The stabilization form $S^{E}: V_{h} \times V_{h} \rightarrow \mathbb{R}$ in (14) must statisfy the following property:

$$
S^{E}\left(v_{h}, v_{h}\right) \sim\left\|\nabla v_{h}\right\|_{E}^{2} \quad \forall v_{h} \in \operatorname{ker} \Pi_{k}^{\nabla} .
$$

A possible choice for $S^{E}$ is

$$
S^{E}\left(v_{h}, w_{h}\right)=\sum_{i=1}^{N_{E}} \chi_{i}\left(v_{h}\right) \chi_{i}\left(w_{h}\right),
$$

where $N_{E}$ is the number of degrees of freedom on the element $E$ and $\chi_{i}$ is the operator that selects the $i$-th degree of freedom.

With the above definitions we can state a SUPG-stabilized discrete formulation of (2) as: find $u_{h} \in V_{h}$ such that

$$
B_{\text {supg }, h}\left(u_{h}, v_{h}\right)=F_{5} \operatorname{supg}, h_{5}\left(v_{h}\right) \quad \forall v_{h} \in V_{h},
$$


having defined the discrete right-hand-side as

$$
F_{\text {supg }, h}\left(v_{h}\right)=\left(f, \Pi_{k-1}^{0} v_{h}\right)+\sum_{E \in \mathcal{T}_{h}} \tau_{E}\left(f, \beta \cdot \Pi_{k-1}^{0} \nabla v_{h}\right)_{E} .
$$

Finally, in order to provide an estimation of the constant $\tilde{C}_{k}^{E}$ for each polygon, we can make use of classical theoretical results on triangles [31] thanks to the following proposition.

Proposition 1. Given a regular polygon $E \in \mathcal{T}_{h}$, let $\mathcal{T}_{h, E}$ be a triangulation of $E$ composed by triangular elements with an edge on the boundary of $E$ and one vertex in the centre of the ball with respect to which $E$ is star-shaped. Let $\tilde{C}_{k}^{E}$ be the constant of inequality (9). Then,

$$
\tilde{C}_{k}^{E} \geq \tilde{C}_{k}\left(\frac{\min _{t \in \mathcal{T}_{h, E}} h_{t}}{h_{E}}\right)^{2}
$$

where $\tilde{C}_{k}$ is such that, $\forall v_{h} \in V_{h}^{E}$,

$$
\tilde{C}_{k} h_{t}^{2}\left\|\nabla \cdot\left(\mathrm{K} \nabla v_{h}\right)\right\|_{t}^{2} \leq\left\|\mathrm{K} \nabla v_{h}\right\|_{t}^{2} \quad \forall t \in \mathcal{T}_{h, E} .
$$

Proof. Summing up the inequalities on internal triangles we have

$$
\tilde{C}_{k} \sum_{t \in \mathcal{T}_{h, E}} h_{t}^{2}\left\|\nabla \cdot\left(\mathrm{K} \nabla v_{h}\right)\right\|_{t}^{2} \leq \sum_{t \in \mathcal{T}_{h, E}}\left\|\mathrm{~K} \nabla v_{h}\right\|_{t}^{2},
$$

from which it follows

$$
\tilde{C}_{k}\left(\min _{t \in \mathcal{T}_{h, E}} h_{t}^{2}\right)\left\|\nabla \cdot\left(\mathrm{K} \nabla v_{h}\right)\right\|_{E}^{2} \leq\left\|\mathrm{K} \nabla v_{h}\right\|_{E}^{2},
$$

and therefore

$$
\tilde{C}_{k}\left(\frac{\min _{t \in \mathcal{T}_{h, E}} h_{t}}{h_{E}}\right)^{2} h_{E}^{2}\left\|\nabla \cdot\left(\mathrm{K} \nabla v_{h}\right)\right\|_{E}^{2} \leq\left\|\mathrm{K} \nabla v_{h}\right\|_{E}^{2},
$$

which proves the thesis.

\section{Error Analysis}

Let $h:=\max _{E \in \mathcal{T}_{h}} h_{E}$ and define the following norm:

$$
\|v\|:=\left\{\|\sqrt{\mathrm{K}} \nabla v\|^{2}+\sum_{E \in \mathcal{T}_{h}} \tau_{E}\|\beta \cdot \nabla v\|_{E}^{2}\right\}^{\frac{1}{2}} \quad \forall v \in \mathrm{H}_{0}^{1}(\Omega) .
$$

In the following, we will use the symbol $\lesssim$ for inequalities which are satisfied up to a multiplicative constant independent of the meshsize and the problem data $\mathrm{K}$ and $\beta$, and the symbol $\precsim$ for inequalities satisfied up to a multiplicative constant independent of the meshsize only. All the constants may depend on the regularity of the VEM mesh. 


\subsection{Discretization errors}

The following Lemmas are devoted to estimate the error of approximation of the bilinear forms defined by (4), (5) and (6) with the discrete ones defined by (14), (15) and (16), respectively. The results are based on the following approximation results for polynomial projections (see [2, Lemma 5.1]): $\forall E \in \mathcal{T}_{h}$,

$$
\begin{gathered}
\left\|v-\Pi_{k-1}^{0} v\right\|_{m, E} \lesssim h_{E}^{s-m}|v|_{s, E} \quad \forall v \in \mathrm{H}^{s}(E), m \leq s \leq k, \\
\left\|v-\Pi_{k}^{\nabla} v\right\|_{m, E} \lesssim h_{E}^{s-m}|v|_{s, E} \quad \forall v \in \mathrm{H}^{s}(E), m \leq s \leq k+1, s \geq 1 .
\end{gathered}
$$

Lemma 1. For any sufficiently regular function $w$ and $\forall v_{h} \in V_{h}$,

$$
b_{h}\left(w, v_{h}\right) \lesssim \max _{E \in \mathcal{T}_{h}} \frac{\beta_{E}}{\sqrt{\mathrm{K}_{E}^{\vee}}}\|\sqrt{\mathrm{K}} \nabla w\|\left\|v_{h}\right\|
$$

Moreover, if $\beta \in\left[\mathrm{W}_{\infty}^{s}(\Omega)\right]^{2}$ for some $s \in\{0, \ldots, k\}$, then

$$
\left|b\left(w, v_{h}\right)-b_{h}\left(w, v_{h}\right)\right| \lesssim \max _{E \in \mathcal{T}_{h}}\|\beta\|_{\mathrm{W}_{\infty}^{s}(E)} h^{s+1}\|w\|_{s+1}\left\|v_{h}\right\|_{1} .
$$

Proof. Regarding 23 , by the Cauchy-Schwarz inequality and the continuity of $\Pi_{k-1}^{0}$ and $\Pi_{k}^{0}$ we have, $\forall E \in \mathcal{T}_{h}$,

$$
\left(\beta \cdot \Pi_{k-1}^{0} \nabla w, \Pi_{k-1}^{0} v_{h}\right)_{E} \leq \beta_{E}\left\|\Pi_{k-1}^{0} \nabla w\right\|_{E}\left\|\Pi_{k-1}^{0} v_{h}\right\|_{E} \lesssim \frac{\beta_{E}}{\sqrt{\mathrm{K}_{E}^{V}}}\|\sqrt{\mathrm{K}} \nabla w\|_{E}\left\|v_{h}\right\|_{E}
$$

from which 23 readily follows.

Concerning (24), let $E \in \mathcal{T}_{h}$ be fixed. By adding and subtracting $\left(\beta \cdot \Pi_{k-1}^{0} \nabla w, v_{h}\right)_{E}$ in the left-hand side and using the triangle inequality,

$$
\begin{aligned}
& \left|\left(\beta \cdot \nabla w, v_{h}\right)_{E}-\left(\beta \cdot \Pi_{k-1}^{0} \nabla w, \Pi_{k-1}^{0} v_{h}\right)_{E}\right|= \\
& =\left|\left(\beta \cdot\left(\nabla w-\Pi_{k-1}^{0} \nabla w\right), v_{h}\right)_{E}+\left(\beta \cdot \Pi_{k-1}^{0} \nabla w, v_{h}-\Pi_{k-1}^{0} v_{h}\right)_{E}\right| \leq \\
& \quad \leq\left|\left(\beta \cdot\left(\nabla w-\Pi_{k-1}^{0} \nabla w\right), v_{h}\right)_{E}\right|+\left|\left(\beta \cdot \Pi_{k-1}^{0} \nabla w, v_{h}-\Pi_{k-1}^{0} v_{h}\right)_{E}\right| .
\end{aligned}
$$

We consider the two terms in the sum separately. The first one can be written as

$$
\left(\beta \cdot\left(\nabla w-\Pi_{k-1}^{0} \nabla w\right), v_{h}\right)_{E}=\sum_{i=1}^{2}\left(\frac{\partial w}{\partial x_{i}}-\Pi_{k-1}^{0} \frac{\partial w}{\partial x_{i}}, \beta_{i} v_{h}\right)_{E} .
$$

Estimating each term in the right-hand side we have, $\forall i \in\{1,2\}$,

$$
\begin{gathered}
\left(\frac{\partial w}{\partial x_{i}}-\Pi_{k-1}^{0} \frac{\partial w}{\partial x_{i}}, \beta_{i} v_{h}\right)_{E}=\left(\frac{\partial w}{\partial x_{i}}-\Pi_{k-1}^{0} \frac{\partial w}{\partial x_{i}}, \beta_{i} v_{h}-\Pi_{k-1}^{0}\left(\beta_{i} v_{h}\right)\right)_{E} \leq \\
\leq\left\|\frac{\partial w}{\partial x_{i}}-\Pi_{k-1}^{0} \frac{\partial w}{\partial x_{i}}\right\|_{E}\left\|\beta_{i} v_{h}-\Pi_{k-1}^{0}\left(\beta_{i} v_{h}\right)\right\|_{E} \lesssim h_{E}^{s}|w|_{s+1, E} \cdot h_{E}\left|\beta_{i} v_{h}\right|_{1, E} \leq \\
\leq h_{E}^{s+1}\left\|\beta_{i}\right\|_{\mathrm{W}_{\infty}^{1}(E)}|w|_{s+1, E}\left\|v_{h}\right\|_{1, E} .
\end{gathered}
$$


Concerning the second term, we have that

$$
\left(\beta \cdot \Pi_{k-1}^{0} \nabla w, v_{h}-\Pi_{k-1}^{0} v_{h}\right)_{E}=\sum_{i=1}^{2}\left(\beta_{i} \Pi_{k-1}^{0} \frac{\partial w}{\partial x_{i}}, v_{h}-\Pi_{k-1}^{0} v_{h}\right)_{E} .
$$

Thus, using the properties of projectors to add polynomials of degree less or equal than $k-1$, we have

$$
\begin{aligned}
&\left(\beta_{i} \Pi_{k-1}^{0} \frac{\partial w}{\partial x_{i}}, v_{h}-\Pi_{k-1}^{0} v_{h}\right)_{E}= \\
&=\left(\beta_{i} \Pi_{k-1}^{0} \frac{\partial w}{\partial x_{i}}-\Pi_{k-1}^{0}\left(\beta_{i} \Pi_{k-1}^{0} \frac{\partial w}{\partial x_{i}}\right), v_{h}-\Pi_{k-1}^{0} v_{h}\right)_{E} \leq \\
& \leq\left\|\beta_{i} \Pi_{k-1}^{0} \frac{\partial w}{\partial x_{i}}-\Pi_{k-1}^{0}\left(\beta_{i} \Pi_{k-1}^{0} \frac{\partial w}{\partial x_{i}}\right)\right\|_{E}\left\|v_{h}-\Pi_{k-1}^{0} v_{h}\right\|_{E} \leq \\
& \leq h_{E}\left\|\beta_{i} \Pi_{k-1}^{0} \frac{\partial w}{\partial x_{i}}-\Pi_{k-1}^{0}\left(\beta_{i} \Pi_{k-1}^{0} \frac{\partial w}{\partial x_{i}}\right)\right\|_{E}\left\|\nabla v_{h}\right\|_{E},
\end{aligned}
$$

and the proof ends using the best approximation property of the projection, the triangle inequality and 21):

$$
\begin{aligned}
&\left\|\beta_{i} \Pi_{k-1}^{0} \frac{\partial w}{\partial x_{i}}-\Pi_{k-1}^{0}\left(\beta_{i} \Pi_{k-1}^{0} \frac{\partial w}{\partial x_{i}}\right)\right\|_{E} \leq \\
& \leq\left\|\beta_{i} \Pi_{k-1}^{0} \frac{\partial w}{\partial x_{i}}-\Pi_{k-1}^{0}\left(\beta_{i} \frac{\partial w}{\partial x_{i}}\right)\right\|_{E} \leq\left\|\beta_{i} \Pi_{k-1}^{0} \frac{\partial w}{\partial x_{i}}-\beta_{i} \frac{\partial w}{\partial x_{i}}\right\|_{E} \\
&+\left\|\beta_{i} \frac{\partial w}{\partial x_{i}}-\Pi_{k-1}^{0}\left(\beta_{i} \frac{\partial w}{\partial x_{i}}\right)\right\|_{E} \leq h_{E}^{s} \beta_{E}|w|_{s+1, E}+h_{E}^{s}\left|\beta_{i} \frac{\partial w}{\partial x_{i}}\right|_{s, E} \leq \\
& \leq h_{E}^{s}\left(\beta_{E}|w|_{s+1, E}+\left\|\beta_{i}\right\|_{\mathrm{W}_{\infty}^{s}(E)}\|w\|_{s+1, E}\right) .
\end{aligned}
$$

Lemma 2. For any sufficiently regular function $w$ and $\forall v_{h} \in V_{h}$,

$$
d_{h}\left(w, v_{h}\right) \lesssim \max _{E \in \mathcal{T}_{h}} \frac{\mathrm{K}_{E}}{\mathrm{~K}_{E}^{V}}\|\sqrt{\mathrm{K}} \nabla w\|\left\|\sqrt{\mathrm{K}} \nabla v_{h}\right\| .
$$

Moreover, if $\mathrm{K} \in \mathrm{W}_{\infty}^{s}(\Omega)$ and $\beta \in\left[\mathrm{W}_{\infty}^{s+1}(\Omega)\right]^{2}$ for some $s \in\{0, \ldots, k\}$, then

$$
\begin{array}{r}
\left|d\left(w, v_{h}\right)-d_{h}\left(w, v_{h}\right)\right| \lesssim \max _{E \in \mathcal{T}_{h}} \frac{\|\beta\|_{\mathrm{W}_{\infty}^{s+1}(E)}\|\mathrm{K}\|_{\mathrm{W}_{\infty}^{s}(E)}\left(\mathrm{K}_{E}+\beta_{E}\right)}{\mathrm{K}_{E} \beta_{E} \sqrt{\mathrm{K}_{E}^{\vee}}} h^{s+1}\|w\|_{s+1} \times \\
\times\left\|\sqrt{\mathrm{K}} \nabla v_{h}\right\| .
\end{array}
$$

Proof. To prove 25), we assume $\nabla \cdot(\mathrm{K} \nabla w) \neq 0$, since otherwhise the inequality is obviously true. We use 12 , the Cauchy-Schwarz inequality, the continuity of $\Pi_{k-1}^{0}$ and 
(9): $\forall E \in \mathcal{T}_{h}$,

$$
\begin{aligned}
\tau_{E}\left(\nabla \cdot\left(\mathrm{K} \Pi_{k-1}^{0} \nabla w\right), \beta \cdot \Pi_{k-1}^{0} \nabla v_{h}\right)_{E} \leq \beta_{E} \frac{h_{E}}{2 \beta_{E}}\left\|\nabla \cdot\left(\mathrm{K} \Pi_{k-1}^{0} \nabla w\right)\right\|_{E} \times \\
\times\left\|\Pi_{k-1}^{0} \nabla v_{h}\right\|_{E} \lesssim \frac{1}{2 \sqrt{\tilde{C}_{E}}}\left\|\mathrm{~K} \Pi_{k-1}^{0} \nabla w\right\|_{E}\left\|\nabla v_{h}\right\|_{E} \lesssim \frac{\mathrm{K}_{E}}{2 \sqrt{\tilde{C}_{E}}} \times \\
\times\left\|\Pi_{k-1}^{0} \nabla w\right\|_{E}\left\|\nabla v_{h}\right\|_{E} \lesssim \frac{\mathrm{K}_{E}}{2 \mathrm{~K}_{E}^{\vee} \sqrt{\tilde{C}_{E}}}\|\sqrt{\mathrm{K}} \nabla w\|_{E}\left\|\sqrt{\mathrm{K}} \nabla v_{h}\right\|_{E} .
\end{aligned}
$$

Regarding 26), by applying the triangle inequality we have:

$$
\begin{gathered}
\left|\sum_{E \in \mathcal{T}_{h}} \tau_{E}\left(\nabla \cdot(\mathrm{K} \nabla w), \beta \cdot \nabla v_{h}\right)_{E}-\tau_{E}\left(\nabla \cdot\left(\mathrm{K} \Pi_{k-1}^{0} \nabla w\right), \beta \cdot \Pi_{k-1}^{0} \nabla v_{h}\right)_{E}\right| \leq \\
\leq \sum_{E \in \mathcal{T}_{h}} \tau_{E}\left|\left(\nabla \cdot\left(\mathrm{K} \nabla w-\mathrm{K} \Pi_{k-1}^{0} \nabla w\right), \beta \cdot \nabla v_{h}\right)_{E}\right| \\
\quad+\tau_{E}\left|\left(\nabla \cdot\left(\mathrm{K} \Pi_{k-1}^{0} \nabla w\right), \beta \cdot\left(\nabla v_{h}-\Pi_{k-1}^{0} \nabla v_{h}\right)\right)_{E}\right| .
\end{gathered}
$$

To estimate the first term of the right-hand-side of $(27)$, we suppose $\nabla \cdot\left(\mathrm{K} \nabla w-\mathrm{K} \Pi_{k-1}^{0} \nabla w\right) \neq$ 0 , we use the Cauchy-Schwarz inequality, (11), (9) and (21):

$$
\begin{gathered}
\tau_{E}\left|\left(\nabla \cdot\left(\mathrm{K} \nabla w-\mathrm{K} \Pi_{k-1}^{0} \nabla w\right), \beta \cdot \nabla v_{h}\right)_{E}\right| \leq \frac{\tilde{C}_{E} h_{E}^{2} \beta_{E}}{2 \mathrm{~K}_{E}}\left\|\nabla \cdot\left(\mathrm{K} \nabla w-\mathrm{K}_{k-1}^{0} \nabla w\right)\right\|_{E} \times \\
\times\left\|\nabla v_{h}\right\|_{E} \leq \frac{\sqrt{\tilde{C}_{E}} h_{E} \beta_{E}}{2 \mathrm{~K}_{E}}\left\|\mathrm{~K} \nabla w-\mathrm{K} \Pi_{k-1}^{0} \nabla w\right\|_{E}\left\|\nabla v_{h}\right\|_{E} \leq \\
\leq \frac{\sqrt{\tilde{C}_{E}} \beta_{E}}{2 \sqrt{\mathrm{K}_{E}^{V}}} h_{E}\left\|\nabla w-\Pi_{k-1}^{0} \nabla w\right\|_{E}\left\|\sqrt{\mathrm{K}} \nabla v_{h}\right\|_{E} \lesssim \frac{\sqrt{\tilde{C}_{E}} \beta_{E}}{2 \sqrt{\mathrm{K}_{E}^{V}}} h_{E}^{s+1}|w|_{s+1, E}\left\|\sqrt{\mathrm{K}} \nabla v_{h}\right\|_{E} .
\end{gathered}
$$

Concerning the second term of (27), we have

$$
\begin{aligned}
\tau_{E}\left(\nabla \cdot\left(\mathrm{K} \Pi_{k-1}^{0} \nabla w\right), \beta \cdot\left(\nabla v_{h}\right.\right. & \left.\left.-\Pi_{k-1}^{0} \nabla v_{h}\right)\right)_{E}= \\
& =\tau_{E} \sum_{i=1}^{2}\left(\beta_{i} \nabla \cdot\left(\mathrm{K} \Pi_{k-1}^{0} \nabla w\right), \frac{\partial v_{h}}{\partial x_{i}}-\Pi_{k-1}^{0}\left(\frac{\partial v_{h}}{\partial x_{i}}\right)\right)_{E},
\end{aligned}
$$

and we can bound each term of the sum by using the properties of the projection, the 
Cauchy-Schwarz inequality and the triangle inequality:

$$
\begin{gathered}
\tau_{E}\left(\beta_{i} \nabla \cdot\left(\mathrm{K} \Pi_{k-1}^{0} \nabla w\right), \frac{\partial v_{h}}{\partial x_{i}}-\Pi_{k-1}^{0} \frac{\partial v_{h}}{\partial x_{i}}\right)_{E}= \\
\tau_{E}\left(\nabla \cdot\left(\beta_{i} \mathrm{~K} \Pi_{k-1}^{0} \nabla w\right), \frac{\partial v_{h}}{\partial x_{i}}-\Pi_{k-1}^{0} \frac{\partial v_{h}}{\partial x_{i}}\right)_{E} \\
+\tau_{E}\left(-\nabla \beta_{i} \cdot\left(\mathrm{K} \Pi_{k-1}^{0} \nabla w\right), \frac{\partial v_{h}}{\partial x_{i}}-\Pi_{k-1}^{0} \frac{\partial v_{h}}{\partial x_{i}}\right)_{E}= \\
=\tau_{E}\left(\nabla \cdot\left(\beta_{i} \mathrm{~K} \Pi_{k-1}^{0} \nabla w\right)-\nabla \cdot\left(\Pi_{k-1}^{0}\left(\beta_{i} \mathrm{~K} \Pi_{k-1}^{0} \nabla w\right)\right), \frac{\partial v_{h}}{\partial x_{i}}-\Pi_{k-1}^{0} \frac{\partial v_{h}}{\partial x_{i}}\right)_{E} \\
+\tau_{E}\left(\Pi_{k-1}^{0}\left(\nabla \beta_{i} \cdot\left(\mathrm{K} \Pi_{k-1}^{0} \nabla w\right)\right)-\nabla \beta_{i} \cdot\left(\mathrm{K} \Pi_{k-1}^{0} \nabla w\right), \frac{\partial v_{h}}{\partial x_{i}}-\Pi_{k-1}^{0} \frac{\partial v_{h}}{\partial x_{i}}\right)_{E} \lesssim \\
\lesssim \frac{\tau_{E}}{\sqrt{\mathrm{K}_{E}^{V}}}\left(\left\|\nabla \cdot\left(\beta_{i} \mathrm{~K} \Pi_{k-1}^{0}(\nabla w)-\Pi_{k-1}^{0}\left(\beta_{i} \mathrm{~K} \Pi_{k-1}^{0} \nabla w\right)\right)\right\|_{E}\right. \\
\left.+\left\|\Pi_{k-1}^{0}\left(\nabla \beta_{i} \cdot\left(\mathrm{K} \Pi_{k-1}^{0} \nabla w\right)\right)-\nabla \beta_{i} \cdot\left(\mathrm{K} \Pi_{k-1}^{0} \nabla w\right)\right\|_{E}\right)\left\|\sqrt{\mathrm{K}} \nabla v_{h}\right\|_{E}
\end{gathered}
$$

We consider the two terms inside the parentheses separately. To estimate the first one, we first use the fact that $\Pi_{k-1}^{0}$ is the best $\mathrm{L}^{2}(E)$ approximation in $\mathbb{P}_{k-1}(E)$, then inequalities (11) and (9), and finally (21):

$$
\begin{gathered}
\tau_{E}\left\|\nabla \cdot\left(\beta_{i} \mathrm{~K} \Pi_{k-1}^{0} \nabla w-\Pi_{k-1}^{0}\left(\beta_{i} \mathrm{~K} \Pi_{k-1}^{0} \nabla w\right)\right)\right\|_{E} \leq \\
\leq \frac{\tilde{C}_{E} h_{E}^{2}}{2 \mathrm{~K}_{E}}\left\|\nabla \cdot\left(\beta_{i} \mathrm{~K} \Pi_{k-1}^{0} \nabla w-\Pi_{k-1}^{0}\left(\beta_{i} \mathrm{~K} \Pi_{k-1}^{0} \nabla w\right)\right)\right\|_{E} \leq \\
\leq \frac{\sqrt{\tilde{C}_{E}} h_{E}}{2 \mathrm{~K}_{E}}\left\|\beta_{i} \mathrm{~K} \Pi_{k-1}^{0} \nabla w-\Pi_{k-1}^{0}\left(\beta_{i} \mathrm{~K} \Pi_{k-1}^{0} \nabla w\right)\right\|_{E} \leq \\
\leq \frac{\sqrt{\tilde{C}_{E}} h_{E}}{2 \mathrm{~K}_{E}}\left\|\beta_{i} \mathrm{~K} \Pi_{k-1}^{0} \nabla w-\Pi_{k-1}^{0}\left(\beta_{i} \mathrm{~K} \nabla w\right)\right\|_{E} \leq \\
\leq \frac{\sqrt{\tilde{C}_{E}} h_{E}}{2 \mathrm{~K}_{E}} \\
\left(\left\|\beta_{i} \mathrm{~K}\left(\Pi_{k-1}^{0} \nabla w-\nabla w\right)\right\|_{E}+\left\|\beta_{i} \mathrm{~K} \nabla w-\Pi_{k-1}^{0}\left(\beta_{i} \mathrm{~K} \nabla w\right)\right\|_{E}\right) \lesssim \\
\lesssim \frac{\sqrt{\tilde{C}_{E}} h_{E}}{2 \mathrm{~K}_{E}}\left(h_{E}^{s} \beta_{E} \mathrm{~K}_{E}|w|_{s+1, E}+h_{E}^{s}\left|\beta_{i} \mathrm{~K} \nabla w\right|_{s, E}\right) \lesssim \\
\lesssim \frac{\sqrt{\tilde{C}_{E}} h_{E}^{s+1}}{2 \mathrm{~K}_{E}}\left(\beta_{E} \mathrm{~K}_{E}|w|_{s+1, E}+\|\beta\|_{\mathrm{W}_{\infty}^{s}(E)}\|\mathrm{K}\|_{\mathrm{W}_{\infty}^{s}(E)}\|w\|_{s+1, E}\right) .
\end{gathered}
$$

To estimate the second term we use the fact that $\Pi_{k-1}^{0}$ is the best approximation in 
$\mathbb{P}_{k-1}(E)$, the triangle inequality, inequality 12 and the estimate 21 :

$$
\begin{aligned}
\tau_{E}\left\|\Pi_{k-1}^{0}\left(\nabla \beta_{i} \cdot\left(\mathrm{K} \Pi_{k-1}^{0} \nabla w\right)\right)-\nabla \beta_{i} \cdot\left(\mathrm{K} \Pi_{k-1}^{0} \nabla w\right)\right\|_{E} \leq \\
\leq \tau_{E}\left\|\Pi_{k-1}^{0}\left(\nabla \beta_{i} \cdot \mathrm{K} \nabla w\right)-\nabla \beta_{i} \cdot \mathrm{K} \Pi_{k-1}^{0} \nabla w\right\|_{E} \leq \frac{h_{E}}{2 \beta_{E}} \times \\
\times\left(\left\|\Pi_{k-1}^{0}\left(\nabla \beta_{i} \cdot \mathrm{K} \nabla w\right)-\nabla \beta_{i} \cdot \mathrm{K} \nabla w\right\|_{E}+\left\|\nabla \beta_{i} \cdot \mathrm{K}\left(\nabla w-\Pi_{k-1}^{0} \nabla w\right)\right\|_{E}\right) \lesssim \\
\lesssim \frac{h_{E}}{2 \beta_{E}}\left(h_{E}^{s}\left|\nabla \beta_{i} \cdot \mathrm{K} \nabla w\right|_{s, E}+h_{E}^{s} \mathrm{~K}_{E}\|\beta\|_{\mathrm{W}_{\infty}^{1}(E)}|w|_{s+1, E}\right) \lesssim \\
\lesssim \frac{h_{E}^{s+1}}{2 \beta_{E}}\left(\|\mathrm{~K}\|_{\mathrm{W}_{\infty}^{s}(E)}\|\beta\|_{\mathrm{W}_{\infty}^{s+1}(E)}\|w\|_{s+1, E}+\mathrm{K}_{E}\|\beta\|_{\mathrm{W}_{\infty}^{1}(E)}|w|_{s+1, E}\right) .
\end{aligned}
$$

Lemma 3. For any sufficiently regular function $w$ and $\forall v_{h} \in V_{h}$,

$$
a_{h}\left(w, v_{h}\right) \lesssim \max _{E \in \mathcal{T}_{h}} \frac{\mathrm{K}_{E}+\tau_{E} \beta_{E}^{2}}{\mathrm{~K}_{E}^{\vee}}\|\sqrt{\mathrm{K}} \nabla w\|\left\|\sqrt{\mathrm{K}} \nabla v_{h}\right\| .
$$

Moreover, if $\mathrm{K} \in \mathrm{W}_{\infty}^{s}(\Omega)$ and $\beta \in\left[\mathrm{W}_{\infty}^{s}(\Omega)\right]$ for some $s \in\{0, \ldots, k\}$, then

$$
\begin{aligned}
&\left|a\left(w, v_{h}\right)-a_{h}\left(w, v_{h}\right)\right| \lesssim\left(\max _{E \in \mathcal{T}_{h}} \frac{\|\mathrm{K}\|_{\mathrm{W}_{\infty}^{s}(E)}+\frac{\|\beta\|_{\mathbf{W}_{\infty}^{s}(E)}^{2}}{\beta_{E}}}{\sqrt{\mathrm{K}_{E}^{V}}}\right) h^{s}\|w\|_{s+1} \times \\
& \times\left\|\sqrt{\mathrm{K}} \nabla v_{h}\right\| .
\end{aligned}
$$

Proof. Let $v_{h}, w \in V_{h}$. We first prove 28 considering $E \in \mathcal{T}_{h}$. Regarding the terms involving the VEM stabilization, we first point out that, as a consequence of (17), we have

$$
S^{E}\left(\left(I-\Pi_{k}^{\nabla}\right) w,\left(I-\Pi_{k}^{\nabla}\right) v_{h}\right) \lesssim\left\|\nabla\left(w-\Pi_{k}^{\nabla} w\right)\right\|_{E}\left\|\nabla\left(v_{h}-\Pi_{k}^{\nabla} v_{h}\right)\right\|_{E} .
$$

Applying the Cauchy-Schwarz inequality, 30 and the continuity of projectors,

$$
\begin{gathered}
a_{h}^{E}\left(w, v_{h}\right)=\left(\mathrm{K}_{k-1}^{0} \nabla w, \Pi_{k-1}^{0} \nabla v_{h}\right)_{E}+\tau_{E}\left(\beta \cdot \Pi_{k-1}^{0} \nabla w, \beta \cdot \Pi_{k-1}^{0} \nabla v_{h}\right)_{E} \\
+\left(\mathrm{K}_{E}+\tau_{E} \beta_{E}^{2}\right) S^{E}\left(\left(I-\Pi_{k}^{\nabla}\right) w,\left(I-\Pi_{k}^{\nabla}\right) v_{h}\right) \lesssim\left(\mathrm{K}_{E}+\tau_{E} \beta_{E}^{2}\right) \times \\
\times\left(\left\|\Pi_{k-1}^{0} \nabla w\right\|_{E}\left\|\Pi_{k-1}^{0} \nabla v_{h}\right\|_{E}+\left\|\left(I-\Pi_{k}^{\nabla}\right) w\right\|_{E}\left\|\left(I-\Pi_{k}^{\nabla}\right) v_{h}\right\|_{E}\right) \lesssim \\
\lesssim \frac{\mathrm{K}_{E}+\tau_{E} \beta_{E}^{2}}{\mathrm{~K}_{E}^{\vee}}\|\sqrt{\mathrm{K}} \nabla w\|_{E}\left\|\sqrt{\mathrm{K}} \nabla v_{h}\right\|_{E} .
\end{gathered}
$$

Concerning (29), by adding and subtracting $\left(\mathrm{K} \nabla w, \Pi_{k-1}^{0} \nabla v_{h}\right)_{E}=\left(\Pi_{k-1}^{0}(\mathrm{~K} \nabla w), \nabla v_{h}\right)_{E}$ and $\left(\beta \beta^{\top} \nabla w, \Pi_{k-1}^{0} \nabla v_{h}\right)_{E}=\left(\Pi_{k-1}^{0}\left(\beta \beta^{\top} \nabla w\right), \nabla v_{h}\right)_{E}$ and exploiting the triangle inequality we have, $\forall E \in \mathcal{T}_{h}$,

$$
\begin{aligned}
& \left|a^{E}\left(w, v_{h}\right)-a_{h}^{E}\left(w, v_{h}\right)\right| \leq\left|\left(\mathrm{K} \nabla w-\mathrm{K} \Pi_{k-1}^{0} \nabla w, \Pi_{k-1}^{0} \nabla v_{h}\right)_{E}\right| \\
& +\left|\left(\mathrm{K} \nabla w-\Pi_{k-1}^{0}(\mathrm{~K} \nabla w), \nabla v_{h}\right)_{E}\right|+\left(\mathrm{K}_{E}+\tau_{E} \beta_{E}^{2}\right) \times \\
& \times\left|S^{E}\left(\left(I-\Pi_{k}^{\nabla}\right) w,\left(I-\Pi_{k}^{\nabla}\right) v_{h}\right)\right|+\tau_{E}\left|\left(\beta \beta^{\top} \nabla w-\beta \beta^{\top} \Pi_{k-1}^{0} \nabla w, \Pi_{k-1}^{0} \nabla v_{h}\right)_{E}\right| \\
& +\tau_{E}\left|\left(\beta \beta^{\top} \nabla w-\Pi_{k-1}^{0}\left(\beta \beta^{\top} \nabla w\right), \nabla v_{h}\right)_{E}\right| .
\end{aligned}
$$


The first term is bounded as follows, exploiting the definition of projection, its continuity and 21):

$$
\begin{aligned}
\left(\mathrm{K}\left(\nabla w-\Pi_{k-1}^{0} \nabla w\right), \Pi_{k-1}^{0} \nabla v_{h}\right)_{E} \lesssim \sqrt{\mathrm{K}_{E}} \| \nabla w & -\Pi_{k-1}^{0} \nabla w\left\|_{E}\right\| \sqrt{\mathrm{K}} \nabla v_{h} \|_{E} \lesssim \\
& \lesssim \sqrt{\mathrm{K}_{E}} h_{E}^{s}|w|_{s+1, E}\left\|\sqrt{\mathrm{K}} \nabla v_{h}\right\|_{E} .
\end{aligned}
$$

The second term is bounded by the Cauchy-Schwarz inequality and 21):

$$
\begin{aligned}
\left(\mathrm{K} \nabla w-\Pi_{k-1}^{0}(\mathrm{~K} \nabla w), \nabla v_{h}\right)_{E} & \leq\left\|\mathrm{K} \nabla w-\Pi_{k-1}^{0}(\mathrm{~K} \nabla w)\right\|_{E}\left\|\nabla v_{h}\right\|_{E} \lesssim \\
& \lesssim h_{E}^{s}|\mathrm{~K} \nabla w|_{s, E} \leq h_{E}^{s} \frac{\|\mathrm{K}\|_{\mathrm{W}_{\infty}^{s}(E)}}{\sqrt{\mathrm{K}_{E}^{V}}}\|w\|_{s+1, E}\left\|\sqrt{\mathrm{K}} \nabla v_{h}\right\| .
\end{aligned}
$$

The third term is estimated using 12 , the Cauchy-Schwarz inequality, the continuity of $\Pi_{k-1}^{0}$ and $(21)$ :

$$
\begin{gathered}
\tau_{E}\left(\beta \beta^{\top}\left(\nabla w-\Pi_{k-1}^{0} \nabla w\right), \Pi_{k-1}^{0} \nabla v_{h}\right)_{E} \lesssim \frac{\beta_{E}}{2} h_{E}\left\|\nabla w-\Pi_{k-1}^{0} \nabla w\right\|_{E}\left\|\nabla v_{h}\right\|_{E} \lesssim \\
\lesssim \frac{\beta_{E}}{2 \sqrt{\mathrm{K}_{E}^{V}}} h_{E}^{s+1}|w|_{s+1, E}\left\|\sqrt{\mathrm{K}} \nabla v_{h}\right\|_{E} \lesssim \frac{\|\beta\|_{\mathrm{W}_{\infty}^{s}(E)}^{2}}{\beta_{E} \sqrt{\mathrm{K}_{E}^{V}}} h_{E}^{s+1}|w|_{s+1, E}\left\|\sqrt{\mathrm{K}} \nabla v_{h}\right\|_{E}
\end{gathered}
$$

The fourth term can be estimated similarly:

$$
\begin{aligned}
& \tau_{E}\left(\beta \beta^{\top} \nabla w-\Pi_{k-1}^{0}\left(\beta \beta^{\top} \nabla w\right), \nabla v_{h}\right)_{E} \leq \tau_{E}\left\|\beta \beta^{\top} \nabla w-\Pi_{k-1}^{0}\left(\beta \beta^{\top} \nabla w\right)\right\|_{E}\left\|\nabla v_{h}\right\| \\
& \quad \lesssim \tau_{E} \frac{h_{E}^{s}}{\sqrt{\mathrm{K}_{E}^{V}}}\left|\beta \beta^{\top} \nabla w\right|_{s, E}\left\|\sqrt{\mathrm{K}} \nabla v_{h}\right\|_{E} \lesssim \frac{\|\beta\|_{\mathrm{W}_{\infty}^{s}(E)}^{2}}{\beta_{E} \sqrt{\mathrm{K}_{E}^{V}}} h_{E}^{s+1}\|w\|_{s+1, E}\left\|\sqrt{\mathrm{K}} \nabla v_{h}\right\|
\end{aligned}
$$

Finally, we consider the terms involving the VEM stabilization and, applying again 30 , we are left to estimate projection errors. Proceeding as above, exploiting the continuity of $\Pi_{k}^{\nabla}, 22$ and the estimate on $\tau_{E}$ in 12 we obtain

$$
\begin{gathered}
\mathrm{K}_{E}\left\|\nabla\left(w-\Pi_{k}^{\nabla} w\right)\right\|_{E}\left\|\nabla\left(v_{h}-\Pi_{k}^{\nabla} v_{h}\right)\right\|_{E} \leq \frac{\mathrm{K}_{E}}{\sqrt{\mathrm{K}_{E}^{V}}} h_{E}^{s}|w|_{s+1, E}\left\|\sqrt{\mathrm{K}} \nabla v_{h}\right\|_{E}, \\
\tau_{E} \beta_{E}^{2}\left\|\nabla\left(w-\Pi_{k}^{\nabla} w\right)\right\|_{E}\left\|\nabla\left(v_{h}-\Pi_{k}^{\nabla} v_{h}\right)\right\|_{E} \leq \frac{\beta_{E}}{2 \sqrt{\mathrm{K}_{E}^{V}}} h_{E}^{s+1}|w|_{s+1, E}\left\|\sqrt{\mathrm{K}} \nabla v_{h}\right\|_{E} .
\end{gathered}
$$

\subsection{Well-posedness of the discrete problem}

In this subsection we prove, in Theorem 1, an inf-sup condition for the discrete bilinear form defined by (13), which ensures the well-posedness of problem (19).

Lemma 4. There exist a constant $\alpha>0$ such that

$$
a_{h}\left(v_{h}, v_{h}\right) \geq \alpha\left\|v_{h}\right\|^{2} \quad \forall v_{h} \in V_{h} .
$$


Proof. Let $v_{h} \in V_{h}$ and fix $E \in \mathcal{T}_{h}$. From the definition of $a_{h}$ in (14) we have

$$
\begin{aligned}
a_{h}^{E}\left(v_{h}, v_{h}\right):= & \left\|\sqrt{\mathrm{K}} \Pi_{k-1}^{0} \nabla v_{h}\right\|_{E}^{2}+\tau_{E}\left\|\beta \cdot \Pi_{k-1}^{0} \nabla v_{h}\right\|_{E}^{2} \\
& +\left(\mathrm{K}_{E}+\tau_{E} \beta_{E}^{2}\right) S^{E}\left(\left(I-\Pi_{k}^{\nabla}\right) v_{h},\left(I-\Pi_{k}^{\nabla}\right) v_{h}\right) .
\end{aligned}
$$

From (17) and the properties of the orthogonal projection, we have that there exists $c^{*}>0$ such that, $\forall E \in \mathcal{T}_{h}$,

$$
S^{E}\left(\left(I-\Pi_{k}^{\nabla}\right) v_{h},\left(I-\Pi_{k}^{\nabla}\right) v_{h}\right) \geq c^{*}\left\|\nabla\left(v_{h}-\Pi_{k}^{\nabla} v_{h}\right)\right\|_{E}^{2} \geq c^{*}\left\|\nabla v_{h}-\Pi_{k-1}^{0} \nabla v_{h}\right\|_{E}^{2},
$$

and then

$$
\begin{aligned}
\left(\mathrm{K}_{E}+\tau_{E} \beta_{E}^{2}\right) S^{E}( & \left.\left(I-\Pi_{k}^{\nabla}\right) v_{h},\left(I-\Pi_{k}^{\nabla}\right) v_{h}\right) \geq \\
& \geq c^{*}\left(\mathrm{~K}_{E}+\tau_{E} \beta_{E}^{2}\right)\left\|\nabla v_{h}-\Pi_{k-1}^{0} \nabla v_{h}\right\|_{E}^{2} \geq \\
\geq c^{*} & \left(\left\|\sqrt{\mathrm{K}}\left(\nabla v_{h}-\Pi_{k-1}^{0} \nabla v_{h}\right)\right\|_{E}^{2}+\tau_{E}\left\|\beta \cdot\left(\nabla v_{h}-\Pi_{k-1}^{0} \nabla v_{h}\right)\right\|_{E}^{2}\right) .
\end{aligned}
$$

The thesis is thus proven choosing $\alpha=\min \left\{c^{*}, 1\right\}$ :

$$
\begin{aligned}
a_{h}^{E}\left(v_{h}, v_{h}\right) \geq & \left\|\sqrt{\mathrm{K}} \Pi_{k-1}^{0} \nabla v_{h}\right\|_{E}^{2}+\sum_{E \in \mathcal{T}_{h}} \tau_{E}\left\|\beta \cdot \Pi_{k-1}^{0} \nabla v_{h}\right\|_{E}^{2} \\
+c^{*} & \left(\left\|\sqrt{\mathrm{K}}\left(\nabla v_{h}-\Pi_{k-1}^{0} \nabla v_{h}\right)\right\|_{E}^{2}+\tau_{E}\left\|\beta \cdot\left(\nabla v_{h}-\Pi_{k-1}^{0} \nabla v_{h}\right)\right\|_{E}^{2}\right) \geq \\
& \min \left\{c^{*}, 1\right\}\left(\left\|\sqrt{\mathrm{K}} \Pi_{k-1}^{0} \nabla v_{h}\right\|^{2}+\left\|\sqrt{\mathrm{K}}\left(\nabla v_{h}-\Pi_{k-1}^{0} \nabla v_{h}\right)\right\|^{2}\right. \\
& \left.+\tau_{E}\left\|\beta \cdot \Pi_{k-1}^{0} \nabla v_{h}\right\|_{E}^{2}+\tau_{E}\left\|\beta \cdot\left(\nabla v_{h}-\Pi_{k-1}^{0} \nabla v_{h}\right)\right\|_{E}^{2}\right) \geq \\
& \geq\left\{c^{*}, 1\right\}\left(\left\|\sqrt{\mathrm{K}} \nabla v_{h}\right\|^{2}+\tau_{E}\left\|\beta \cdot \nabla v_{h}\right\|_{E}^{2}\right) .
\end{aligned}
$$

Lemma 5. Let $q \in \mathrm{H}_{0}^{1}(\Omega)$. Then there exists $q^{*} \in V_{h}$ such that

$$
a_{h}\left(q^{*}, v_{h}\right)=a\left(q, v_{h}\right) \quad \forall v_{h} \in V_{h} .
$$

Moreover,

$$
\begin{gathered}
\left\|q^{*}\right\| \leq \frac{1}{\alpha}\|q\|, \\
\left\|q-q^{*}\right\| \precsim h\|q\|,
\end{gathered}
$$

being $\alpha$ the coercivity constant in 31.

Proof. The proof is formally the same as the one for [2, Lemma 5.6]. 
Lemma 6. For any $v_{h} \in V_{h}$,

$$
B_{\text {supg }}\left(v_{h}, v_{h}\right) \geq \frac{1}{2}\left\|v_{h}\right\|
$$

Proof. Let $v_{h} \in V_{h}$. Since we have homogeneous Dirichlet boundary conditions and $\nabla \cdot \beta=0$, it holds

$$
\left(\beta \cdot \nabla v_{h}, v_{h}\right)=-\frac{1}{2}\left(\nabla \cdot \beta, v_{h}^{2}\right)=0 .
$$

We have, using the definition of $B_{\text {supg }}$, and the Cauchy-Schwarz and Young inequalities and the estimate $(9)$,

$$
\begin{gathered}
B_{\operatorname{supg}}\left(v_{h}, v_{h}\right)=\left\|\sqrt{\mathrm{K}} \nabla v_{h}\right\|^{2}+\sum_{E \in \mathcal{T}_{h}} \tau_{E}\left\|\beta \cdot \nabla v_{h}\right\|_{E}^{2}-\tau_{E}\left(\nabla \cdot\left(\sqrt{\mathrm{K}} \nabla v_{h}\right), \beta \cdot \nabla v_{h}\right)_{E} \geq \\
\geq\left\|\sqrt{\mathrm{K}} \nabla v_{h}\right\|^{2}+\sum_{E \in \mathcal{T}_{h}} \tau_{E}\left\|\beta \cdot \nabla v_{h}\right\|_{E}^{2}-\tau_{E}\left\|\nabla \cdot\left(\sqrt{\mathrm{K}} \nabla v_{h}\right)\right\|_{E}\left\|\beta \cdot \nabla v_{h}\right\|_{E} \geq\left\|\sqrt{\mathrm{K}} \nabla v_{h}\right\|_{E}^{2} \\
+\sum_{E \in \mathcal{T}_{h}} \frac{1}{2} \tau_{E}\left\|\beta \cdot \nabla v_{h}\right\|_{E}^{2}-\frac{1}{2} \tau_{E}\left\|\nabla \cdot\left(\mathrm{K} \nabla v_{h}\right)\right\|_{E}^{2} \geq\left\|\sqrt{\mathrm{K}} \nabla v_{h}\right\|^{2}-\sum_{E \in \mathcal{T}_{h}} \frac{1}{4}\left\|\sqrt{\mathrm{K}} \nabla v_{h}\right\|_{E}^{2} \\
+\frac{1}{2} \tau_{E}\left\|\beta \cdot \nabla v_{h}\right\|_{E}^{2} \geq \frac{3}{4}\left\|\sqrt{\mathrm{K}} \nabla v_{h}\right\|^{2}+\sum_{E \in \mathcal{T}_{h}} \frac{1}{2} \tau_{E}\left\|\beta \cdot \nabla v_{h}\right\|_{E}^{2} \geq \frac{1}{2}\left\|v_{h}\right\|^{2} .
\end{gathered}
$$

Theorem 1. Suppose $\mathrm{K} \in \mathrm{L}^{\infty}(\Omega)$ and $\beta \in\left[\mathrm{W}_{\infty}^{1}(\Omega)\right]^{2}$. Then, $\forall v_{h} \in V_{h}$ and for $h$ sufficiently small,

$$
\sup _{w_{h} \in V_{h}} \frac{B_{\operatorname{supg}, h}\left(v_{h}, w_{h}\right)}{\left\|w_{h}\right\|} \succsim\left\|v_{h}\right\| .
$$

Proof. Let $v_{h} \in V_{h}$ be fixed and let $v_{h}^{*} \in V_{h}$ be the function, whose existence is guaranteed by Lemma 5, such that $a_{h}\left(v_{h}^{*}, w_{h}\right)=a\left(v_{h}, w_{h}\right), \forall w_{h} \in V_{h}$. By definitions (3) and (13), since $a_{h}$ is symmetric, we have, by 34 ,

$$
\begin{aligned}
B_{\mathrm{supg}, h}\left(v_{h}, v_{h}^{*}\right)= & a_{h}\left(v_{h}, v_{h}^{*}\right)+b_{h}\left(v_{h}, v_{h}^{*}\right)+d_{h}\left(v_{h}, v_{h}^{*}\right)=a\left(v_{h}, v_{h}\right)+b_{h}\left(v_{h}, v_{h}^{*}\right) \\
& +d_{h}\left(v_{h}, v_{h}^{*}\right)=B_{\text {supg }}\left(v_{h}, v_{h}\right)+r\left(v_{h}, v_{h}^{*}\right) \geq \frac{1}{2}\left\|v_{h}\right\|^{2}+r\left(v_{h}, v_{h}^{*}\right),
\end{aligned}
$$

where

$$
\begin{aligned}
r\left(v_{h}, v_{h}^{*}\right)= & b_{h}\left(v_{h}, v_{h}^{*}\right)-b\left(v_{h}, v_{h}^{*}\right)+b\left(v_{h}, v_{h}^{*}-v_{h}\right) \\
& +d_{h}\left(v_{h}, v_{h}^{*}\right)-d\left(v_{h}, v_{h}^{*}\right)+d\left(v_{h}, v_{h}^{*}-v_{h}\right) .
\end{aligned}
$$

By Lemmas 1 and 2, the continuity of $b$ and $d$, that can be proven as for 23) and (25), and by 32 and $(33)$, there exists a constant $C_{r}>0$ depending on $\|\mathrm{K}\|_{\mathrm{L}^{\infty}(\mathrm{K})},\|\beta\|_{\mathrm{W}_{\infty}^{1}(\Omega)}$ and on the approximation constants in 21 and $(22)$, such that

$$
\left|r\left(v_{h}, v_{h}^{*}\right)\right| \leq C_{r} h\left\|\sqrt{\mathrm{K}} \nabla v_{h}\right\|\left\|\sqrt{\mathrm{K}} \nabla v_{h}^{*}\right\| \leq C_{r} h\left\|v_{h}\right\|\left\|v_{h}^{*}\right\| \leq C_{r} h\left\|v_{h}\right\|^{2} .
$$


Then, by 32 and 36 the following lower bound holds:

$$
B_{\text {supg }, h}\left(v_{h}, v_{h}\right) \geq \frac{1}{2}\left\|v_{h}\right\|^{2}+r\left(v_{h}, v_{h}^{*}\right) \geq\left(\frac{\alpha}{2}-C_{r} h\right)\left\|v_{h}\right\|\left\|v_{h}^{*}\right\|,
$$

which yields the thesis for

$$
h<\frac{\alpha}{2 C_{r}} .
$$

\subsection{A priori error estimate}

To derive an a priori estimate that shows optimality of the rate of convergence of this SUPG approach, we will use the following estimate on the VEM interpolator (see [2, Lemma 5.1]):

$\forall E \in \mathcal{T}_{h}, \forall \varphi \in \mathrm{H}^{s}(E),\left\|\varphi-\varphi_{I}\right\|_{m, E} \lesssim h_{E}^{s-m}|\varphi|_{s, E}: \forall s, m \in \mathbb{N}, m \leq s \leq k+1, s \geq 2$.

We are now ready to prove the following result.

Theorem 2. Suppose $u \in \mathrm{H}^{s+1}(\Omega), \mathrm{K} \in \mathrm{W}_{\infty}^{s}(\Omega), \beta \in\left[\mathrm{W}_{\infty}^{s+1}(\Omega)\right]^{2}$ for some $s \in$ $\{1, \ldots, k\}$. Then, for $h$ sufficiently small,

$$
\left\|u-u_{h}\right\| \precsim h^{s}\left(\|u\|_{s+1}+\|f\|_{s}\right) .
$$

Proof. First, by the triangle inequality we have

$$
\left\|u-u_{h}\right\|^{2} \leq\left\|u-u_{I}\right\|^{2}+\left\|u_{h}-u_{I}\right\|^{2},
$$

and, by (37),

$$
\begin{aligned}
\left\|u-u_{I}\right\|^{2}=\sum_{E \in \mathcal{T}_{h}} & \left\|\sqrt{\mathrm{K}} \nabla\left(u-u_{I}\right)\right\|_{E}^{2}+\left\|\beta \cdot \nabla\left(u-u_{I}\right)\right\|_{E}^{2} \leq \\
& \leq \sum_{E \in \mathcal{T}_{h}}\left(\mathrm{~K}_{E}+\beta_{E}^{2}\right)\left\|\nabla\left(u-u_{I}\right)\right\|_{E}^{2} \lesssim \sum_{E \in \mathcal{T}_{h}}\left(\mathrm{~K}_{E}+\beta_{E}^{2}\right) h_{E}^{2 s}|u|_{s+1, E}^{2} .
\end{aligned}
$$

We are left to estimate the norm of $e_{h}:=u_{h}-u_{I}$. Since $e_{h} \in V_{h}$, by 35 there exists $w_{h} \in V_{h}$ such that

$$
B_{\text {supg }, h}\left(e_{h}, w_{h}\right) \succsim\left\|e_{h}\right\|\left\|w_{h}\right\| .
$$

Using the exact and discrete problems 100 and 19 ,

$$
\begin{aligned}
& \left\|e_{h}\right\|\left\|w_{h}\right\| \precsim B_{\text {supg }, h}\left(u_{h}-u_{I}, w_{h}\right)=F_{\text {supg }, h}\left(w_{h}\right)-B_{\text {supg }, h}\left(u_{I}, w_{h}\right)= \\
& =F_{\text {supg }, h}\left(w_{h}\right)-F_{\text {supg }}\left(w_{h}\right)+B_{\text {supg }}\left(u, w_{h}\right)-B_{\text {supg }, h}\left(u_{I}, w_{h}\right)=F_{\text {supg }, h}\left(w_{h}\right) \\
& \quad-F_{\text {supg }}\left(w_{h}\right)+B_{\text {supg }, h}\left(u-u_{I}, w_{h}\right)+B_{\text {supg }}\left(u, w_{h}\right)-B_{\text {supg }, h}\left(u, w_{h}\right) .
\end{aligned}
$$

Note that for our choice of the degrees of freedom and stabilization (defined in $(18)$ ), it makes sense to compute $B_{\operatorname{supg}, h}\left(u, w_{h}\right)$ as in (13)- $(16)$, because $u \in \mathrm{H}^{2}(\Omega) \subset C^{0}(\Omega)$ for $\Omega \subset \mathbb{R}^{2}$. If the solution $u$ does not have the regularity for pointwise evaluation, definition (18) for the VEM-stabilization function has to be properly modified. 
The first difference in $(39)$ can be written as:

$$
F_{\text {supg }, h}\left(w_{h}\right)-F_{\text {supg }}\left(w_{h}\right)=\sum_{E \in \mathcal{T}_{h}}\left(f,\left(\Pi_{k-1}^{0}-I\right) w_{h}+\beta \cdot\left(\Pi_{k-1}^{0}-I\right) \nabla w_{h}\right)_{E} .
$$

The first term of the sum in 40 is bounded as follows:

$$
\begin{aligned}
& \left(f,\left(\Pi_{k-1}^{0}-I\right) w_{h}\right)=\left(\left(I-\Pi_{k-1}^{0}\right) f,\left(\Pi_{k-1}^{0}-I\right) w_{h}\right) \leq\left\|f-\Pi_{k-1}^{0} f\right\|_{E} \times \\
& \times\left\|w_{h}-\Pi_{k-1}^{0} w_{h}\right\|_{E} \lesssim h_{E}^{s-1}|f|_{s-1, E} h_{E}\left\|\nabla w_{h}\right\| \leq \\
& \quad \leq \frac{h_{E}^{s}}{\sqrt{\mathrm{K}_{E}^{\mathrm{V}}}}|f|_{s-1, E}\left\|\sqrt{\mathrm{K}} \nabla w_{h}\right\|_{E} \leq \frac{h_{E}^{s}}{\sqrt{\mathrm{K}_{E}^{\mathrm{V}}}}|f|_{s-1, E}\left\|w_{h}\right\|_{E} .
\end{aligned}
$$

The second term of the sum in 40 can be treated as follows:

$$
\begin{gathered}
\left(f, \beta \cdot\left(\Pi_{k-1}^{0}-I\right) \nabla w_{h}\right)_{E}=\sum_{i=1}^{2}\left(\left(\Pi_{k-1}^{0}-I\right)\left(\beta_{i} f\right), \frac{\partial w_{h}}{\partial x_{i}}\right)_{E} \leq \\
\leq \sum_{i=1}^{2}\left\|\left(I-\Pi_{k-1}^{0}\right)\left(\beta_{i} f\right)\right\|_{E}\left\|\frac{\partial w_{h}}{\partial x_{i}}\right\|_{E} \lesssim \frac{h^{s}}{\sqrt{\mathrm{K}_{E}^{V}}} \sum_{i=1}^{2}\left|\beta_{i} f\right|_{s, E}\left\|\sqrt{\mathrm{K}} \nabla w_{h}\right\|_{E} \leq \\
\leq \frac{\|\beta\|_{\mathrm{W}_{\infty}^{s}(E)}}{\sqrt{\mathrm{K}_{E}^{\vee}}} h_{E}^{s}\|f\|_{s, E}\left\|w_{h}\right\|_{E} .
\end{gathered}
$$

Going back to (39), we estimate the continuity of $B_{\text {supg, },}$, given by 23), 25) and 28), and the estimate on the VEM interpolator in (37):

$$
B_{\text {supg }, h}\left(u-u_{I}, w_{h}\right) \precsim\left\|u-u_{I}\right\|_{1}\left\|w_{h}\right\|_{1} \lesssim h^{s}\|u\|_{s+1}\left\|w_{h}\right\| .
$$

The estimate of the last difference in (39) is obtained by applying (24), 26) and 29):

$$
\begin{aligned}
& \left|B_{\operatorname{supg}}\left(u, w_{h}\right)-B_{\text {supg }, h}\left(u, w_{h}\right)\right| \leq\left|a\left(u, w_{h}\right)-a_{h}\left(u, w_{h}\right)\right| \\
& \quad+\left|b\left(u, w_{h}\right)-b_{h}\left(u, w_{h}\right)\right|+\left|d\left(u, w_{h}\right)-d_{h}\left(u, w_{h}\right)\right| \precsim h^{s}\|u\|_{s+1}\left\|w_{h}\right\|_{1} .
\end{aligned}
$$

\section{Numerical Results}

In this section we will consider two benchmark problems in the domain $\Omega=(0,1) \times$ $(0,1)$ in order to numerically evaluate the rates of convergence of the discussed VEMSUPG stabilization both in the convection-dominated regime and the diffusion-dominated regime. VEM orders from one to three are used.

\subsection{Test 1}

As a first test we consider problem (1) with constant $\mathrm{K}$ and $\beta$. In particular the transport velocity field is

$$
\beta(x, y)=\left(\frac{1}{2},-\frac{1}{3}\right),
$$


and we perform two sets of simulations corresponding to two different values of $\mathrm{K}$ : in a first set of simulations we use $\mathrm{K}=10^{-3}$, whereas $\mathrm{K}=10^{-9}$ is used for a second set of simulations. The meshsize range is chosen in such a way that for all the values of the VEM order $k$ the mesh Péclet number is both greater and lower than one for $\mathrm{K}=10^{-3}$, whereas it is much greater than one for $\mathrm{K}=10^{-9}$.

The exact solution for this problem is given by

$$
u(x, y)=\frac{65536}{729} x^{3}(1-x) y^{3}(1-y) .
$$

In Figures $1 \mathrm{a}$ 1f we show the convergence curves obtained with $\mathrm{K}=10^{-3}$ (left) and $\mathrm{K}=10^{-9}$ (right). The error reported is based on the difference between the exact solution and the projection of the discrete solution on the space of polynomials of degree $k$, accordingly to the VEM order $k$ varying from 1 to 3 . The error is measured in the $\mathrm{L}^{2}(\Omega)$ and $\mathrm{H}^{1}(\Omega)$-norms and is plotted with respect to the number of degrees of freedom (Ndof). For each mesh we also report the values of the minimum and maximum mesh Péclet numbers. Note that the left $y$-axes scales refer to the mesh Péclet numbers, whereas the right ones refer to the error measure. The very good agreement between the numerical behaviour and the expected rates of convergence in $(38)$ is evident.

\subsection{Test 2}

For the second test, non-constant coefficients are used and the flow regime is transport dominated in all the simulations performed. We have set:

$$
\begin{gathered}
\mathrm{K}(x, y)=10^{-7}\left(\begin{array}{cc}
1+x^{2} & x y \\
x y & 1+y^{2}
\end{array}\right), \\
\beta(x, y)=\left(\frac{1}{3}+10 y\left(x+y^{2}\right)^{4},-\frac{1}{2}-5\left(x+y^{2}\right)^{4}\right),
\end{gathered}
$$

and the exact solution in this case is:

$$
u(x, y)=600 x y(1-x)(1-y)\left(x-\frac{1}{5}\right)\left(y-\frac{2}{5}\right)\left(y-\frac{3}{5}\right) .
$$

We now compare the solution obtained with the VEM-SUPG method described in the present work on a family of polygonal Voronoi meshes generated by PolyMesher [32, made up of polygons with four to eight edges (see Figure 2a), with the solution obtained on standard triangular meshes. Figures $2 \mathrm{C}$ and $2 \mathrm{~d}$ show a comparison between the unstabilized solution and the one obtained using the SUPG stabilization for second order VEM, showing a very good agreement with the exact solution (Figure 2b) for a given polygonal mesh. Convergence curves were obtained for VEM formulations of order from 1 to 3 and are reported in Figure 3 . The error was obtained by comparing the exact solution to the polynomial projections of the discrete solutions. On each plot we also report the maximum and minimum mesh Péclet number for each considered meshsize. Also in this case, the left $y$-axes refer to the mesh Péclet numbers, whereas the right ones refer to the error measure. Note that for all orders and meshes, this problem is always convection-dominant $\left(\min _{E \in \mathcal{T}_{h}} \mathrm{Pe}_{E} \gg 1\right.$ for all meshes). Again, the plots show a very good agreement between the experimental orders of convergence and the ones provided by Theorem 2 , independently of the mesh used. 


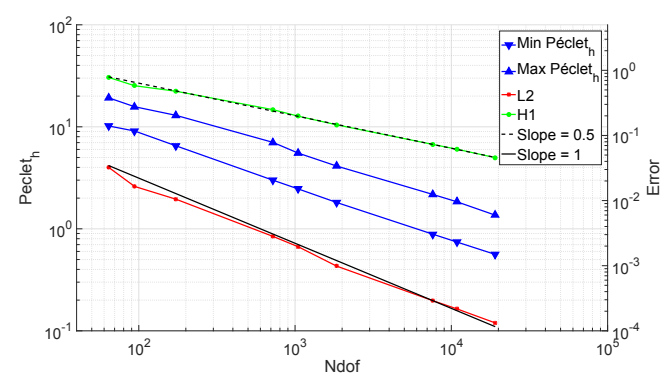

(a) Order $1, \mathrm{~K}=10^{-3}$

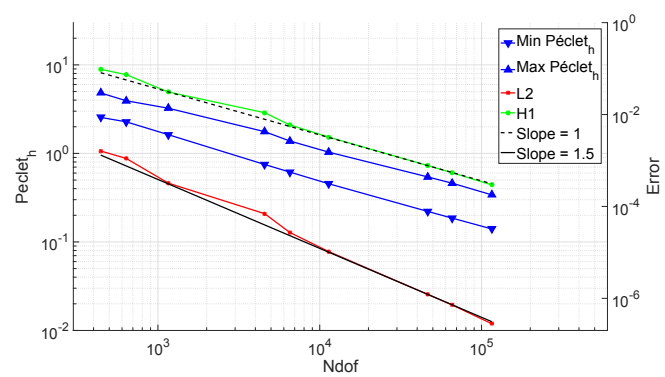

(c) Order $2, \mathrm{~K}=10^{-3}$

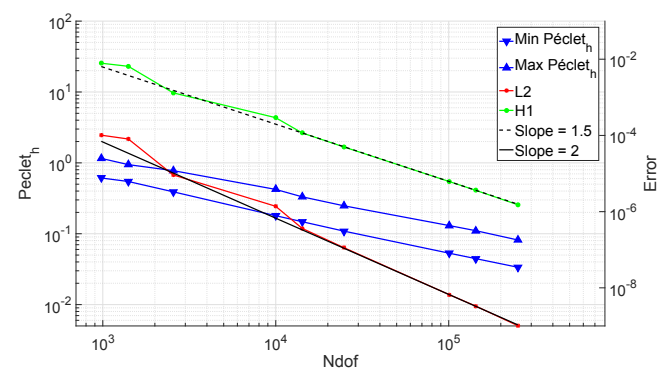

(e) Order $3, \mathrm{~K}=10^{-3}$

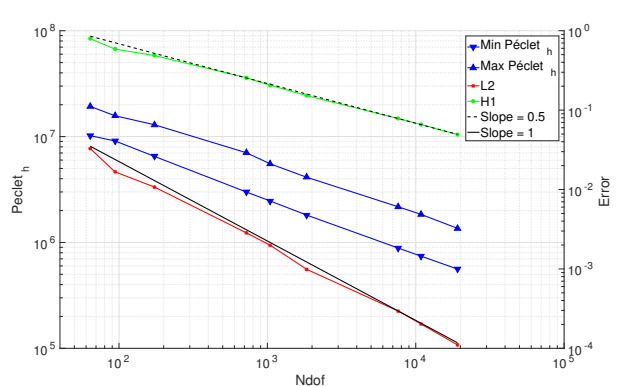

(b) Order $1, \mathrm{~K}=10^{-9}$

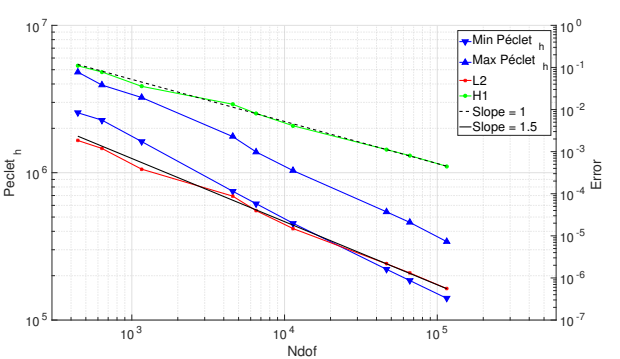

(d) Order $2, \mathrm{~K}=10^{-9}$

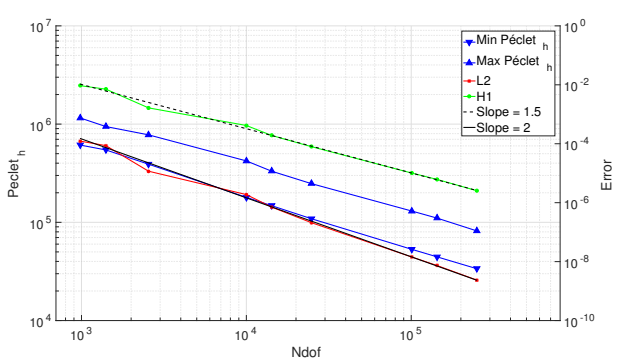

(f) Order $3, \mathrm{~K}=10^{-9}$

Figure 1: Test 1. Convergence curves 


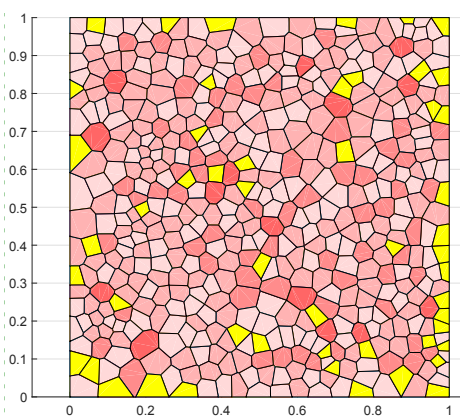

(a) Sample random polygonal mesh

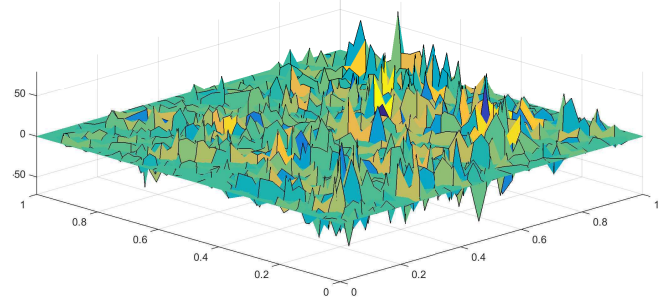

(c) Unstabilized solution

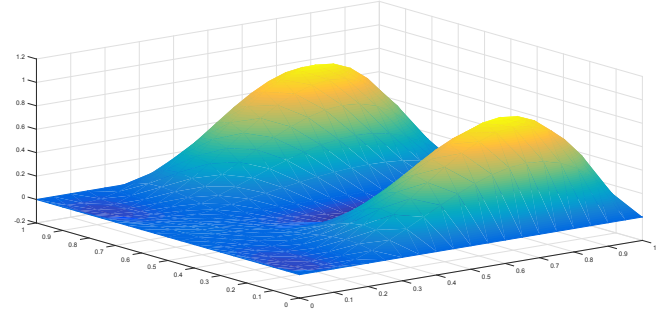

(b) Exact solution

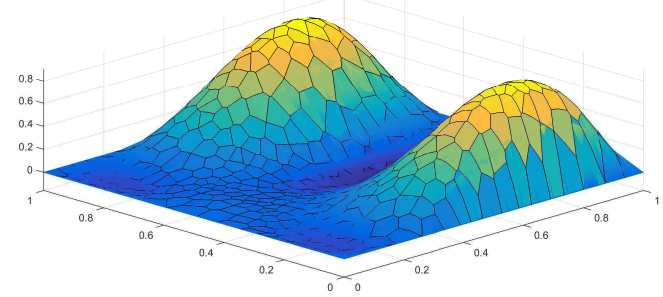

(d) Stabilized solution

Figure 2: Test 2. Sample mesh, exact, unstabilized and stabilized solutions

\section{Conclusions}

In this paper we have considered the advection-diffusion problem with a VEM based approach. The stabilization considered is a natural extension to the VEM of the classical SUPG stabilization for the standard FEM. It is known from the VEM literature that VEM discretizations require the introduction of a stabilization term to ensure coercivity of the discrete operators. A VEM stabilization of the SUPG stabilization is therefore needed. Under sufficient regularity assumptions of the data and of the exact solution, we have shown that both the advective-SUPG stabilization and the corresponding VEM stabilization for coercivity (stabilization of a stabilization) do not pollute the rates of convergence of the VEM discretization.

Numerical results confirm the proven theoretical behaviour. Moreover, stable good discrete solutions are obtained also for very large Péclet numbers in the order of $10^{9}$ and mesh Péclet numbers in the order of $10^{7}$. The numerical results also show a reliable stabilizing effect for the proposed formulation of the SUPG stabilization without the introduction of an excessive diffusive effect.

\section{References}

[1] L. Beirão da Veiga, F. Brezzi, A. Cangiani, G. Manzini, L. D. Marini, A. Russo, Basic principles of virtual element methods, Mathematical Models and Methods in Applied Sciences 23 (01) (2013) 199-214.

[2] L. Beirão da Veiga, F. Brezzi, L. D. Marini, A. Russo, Virtual element methods for general second order elliptic problems on polygonal meshes, Mathematical Models and Methods in Applied Sciences 26 (04) (2015) 729-750. doi:10.1142/S0218202516500160 


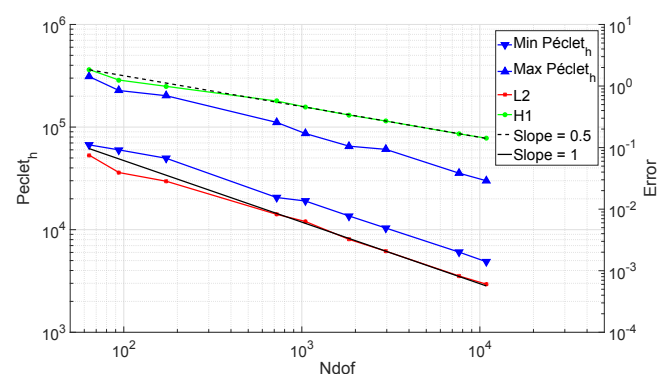

(a) Order 1, triangular mesh

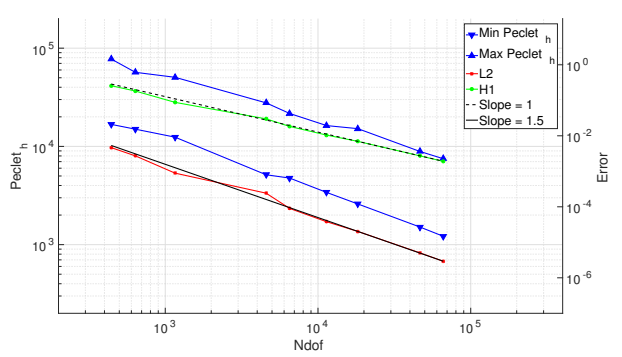

(c) Order 2, triangular mesh

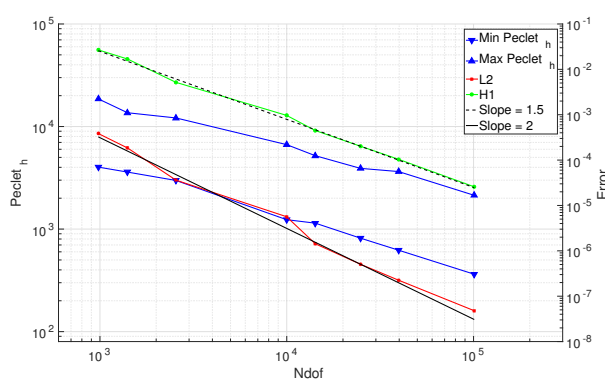

(e) Order 3, triangular mesh

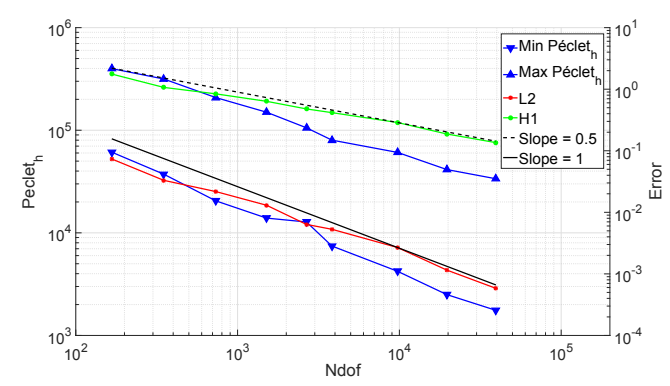

(b) Order 1, polygonal mesh

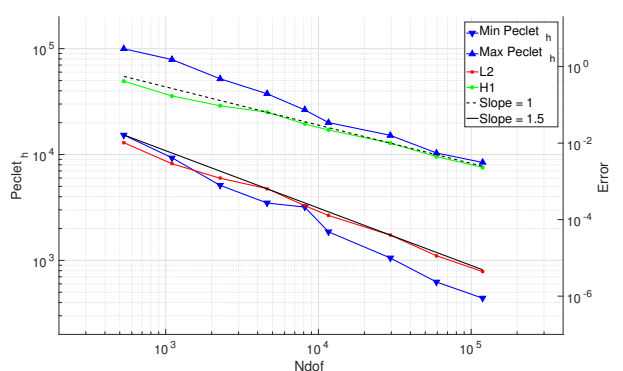

(d) Order 2, polygonal mesh

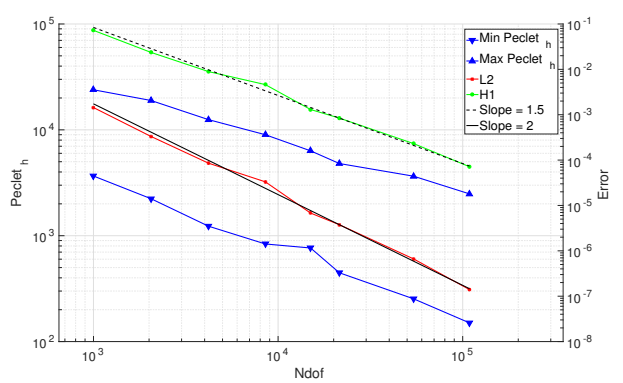

(f) Order 3, polygonal mesh

Figure 3: Test 2. Convergence curves 
[3] F. Brezzi, L. D. Marini, Virtual element methods for plate bending problems, Computer Methods in Applied Mechanics and Engineering 253 (2013) 455-462. doi:http://dx.doi.org/10.1016/j. cma.2012.09.012

[4] L. Beirão da Veiga, F. Brezzi, L. D. Marini, Virtual elements for linear elasticity problems, SIAM Journal on Numerical Analysis 51 (2) (2013) 794-812. doi:10.1137/120874746

[5] L. Beirão da Veiga, C. Lovadina, D. Mora, A virtual element method for elastic and inelastic problems on polytope meshes, Computer Methods in Applied Mechanics and Engineering 295 (2015) 327 - 346. doi:http://dx.doi.org/10.1016/j.cma.2015.07.013

[6] P. F. Antonietti, L. Beirão da Veiga, D. Mora, M. Verani, A stream virtual element formulation of the stokes problem on polygonal meshes, SIAM Journal on Numerical Analysis 52 (1) (2014) 386-404. doi:10.1137/13091141X

[7] D. Mora, G. Rivera, R. Rodríguez, A virtual element method for the Steklov eigenvalue problem, Mathematical Models and Methods in Applied Sciences 25 (08) (2015) 1421-1445. doi:10.1142/ S0218202515500372

[8] A. Cangiani, G. Manzini, O. J. Sutton, Conforming and nonconforming virtual element methods for elliptic problems, available online at http://arxiv.org/abs/1507.03543 (2015).

[9] M. Benedetto, S. Berrone, S. Pieraccini, S. Scialò, The virtual element method for discrete fracture network simulations, Comput. Methods Appl. Mech. Engrg. 280 (0) (2014) 135 - 156. doi:http: //dx.doi.org/10.1016/j.cma.2014.07.016

[10] M. Benedetto, S. Berrone, S. Scialò, A globally conforming method for solving flow in discrete fracture networks using the virtual element method, Finite Elem. Anal. Des. 109 (2016) 23-36. doi:http://dx.doi.org/10.1016/j.finel.2015.10.003

[11] M. Benedetto, S. Berrone, A. Borio, S. Pieraccini, S. Scialò, A hybrid mortar virtual element method for discrete fracture network simulations, J. Comput. Phys. 306 (2016) 148-166. doi : http: //dx.doi.org/10.1016/j.jcp.2015.11.034

[12] S. Ganesan, L. Tobiska, Stabilization by local projection for convection - diffusion and incompressible flow problems, Journal of Scientific Computing 43 (3) (2010) 326-342.

[13] F. Brezzi, L. Franca, A. Russo, Further considerations on residual-free bubbles for advective-diffusive equations, Computer Methods in Applied Mechanics and Engineering 166 (1 - 2) (1998) 25 - 33 , advances in Stabilized Methods in Computational Mechanics. doi:http://dx.doi.org/10.1016/ S0045-7825(98) 00080-2

[14] L. P. Franca, L. Tobiska, Stability of the residual free bubble method for bilinear finite elements on rectangular grids, IMA journal of numerical analysis 22 (1) (2002) 73-87.

[15] A. N. Brooks, T. J. R. Hughes, Streamline Upwind / Petrov-Galerkin formulation for convective dominated flows with particular emphasis on the incompressible navier - stokes equations, Comput. Methods Appl. Engrg. 32 (1982) 199 - 259.

[16] C. Johnson, U. Nävert, J. Pitkäranta, Finite element methods for linear hyperbolic problems, Computer Methods in Applied Mechanics and Engineering 45 (1984) 285 - 312. doi:10.1016/ 0045-7825(84)90158-0

[17] L. P. Franca, S. L. Frey, T. J. Hughes, Stabilized finite element methods: I. Application to the advective-diffusive model, Computer Methods in Applied Mechanics and Engineering 95 (2) (1992) 253 - 276. doi:http://dx.doi.org/10.1016/0045-7825(92)90143-8

[18] T. Gelhard, G. Lube, M. A. Olshanskii, J.-H. Starcke, Stabilized finite element schemes with LBB - stable elements for incompressible flows, Journal of Computational and Applied Mathematics 177 (2) (2005) 243 - 267. doi:http://dx.doi.org/10.1016/j.cam.2004.09.017

[19] H. G. Roos, M. Stynes, L. Tobiska, Robust numerical methods for singularly perturbed differential equations: convection-diffusion-reaction and flow problems, Vol. 24, Springer Science \& Business Media, 2008.

[20] E. Burman, Consistent SUPG-method for transient transport problems: Stability and convergence, Computer Methods in Applied Mechanics and Engineering 199 (17 - 20) (2010) 1114 - 1123. doi:http://dx.doi.org/10.1016/j.cma.2009.11.023

[21] E. Burman, G. Smith, Analysis of the space semi-discretized SUPG method for transient convectiondiffusion equations, Mathematical Models and Methods in Applied Sciences 21 (10) (2011) 20492068.

[22] A. Cangiani, G. Manzini, O. Sutton, The conforming virtual element method for the convectiondiffusion-reaction equation with variable coefficients, Techical Report, Los Alamos National Laboratory, available online at http://www.osti.gov/scitech/servlets/purl/1159207 (2014).

[23] S. Berrone, Adaptive discretization of stationary and incompressible Navier-Stokes equations by stabilized finite element methods, Computer Methods in Applied Mechanics and Engineering 190 (34) 
(2001) 4435 - 4455. doi:http://dx.doi.org/10.1016/S0045-7825(00)00327-3

[24] S. Berrone, Robustness in a posteriori error analysis for fem flow models, Numerische Mathematik 91 (3) (2002) 389-422. doi:10.1007/s002110100370.

[25] R. Verfürth, Robust a posteriori error estimates for stationary convection-diffusion equations, SIAM Journal on Numerical Analysis 43 (4) (2005) 1766-1782. doi:10.1137/040604261

[26] R. Verfürth, Robust a posteriori error estimates for nonstationary convection-diffusion equations, SIAM Journal on Numerical Analysis 43 (4) (2005) 1783-1802. doi:10.1137/040604273

[27] S. Berrone, M. Marro, Space-time adaptive simulations for unsteady Navier-Stokes problems, Computers \& Fluids 38 (6) (2009) 1132 - 1144. doi:http://dx.doi.org/10.1016/j.compfluid.2008. 11.004

[28] S. Berrone, M. Marro, Numerical investigation of effectivity indices of space-time error indicators for Navier-Stokes equations, Computer Methods in Applied Mechanics and Engineering 199 (2528) (2010) 1764 - 1782. doi:http://dx.doi.org/10.1016/j.cma.2010.02.004

[29] A. Cangiani, E. H. Georgoulis, T. Pryer, O. J. Sutton, A posteriori error estimates for the virtual element method, available online at http://adsabs.harvard.edu/abs/2016arXiv160305855C (2016).

[30] L. Beirão Da Veiga, F. Brezzi, L. D. Marini, A. Russo, The hitchhiker's guide to the virtual element method, Math. Models Methods Appl. Sci 24 (8) (2014) 1541-1573.

[31] I. Harari, T. J. Hughes, What are $\mathrm{C}$ and h?: Inequalities for the analysis and design of finite element methods, Computer Methods in Applied Mechanics and Engineering 97 (2) (1992) 157 192. doi:http://dx.doi.org/10.1016/0045-7825(92)90162-D

[32] C. Talischi, G. H. Paulino, A. Pereira, I. F. M. Menezes, Polymesher: a general-purpose mesh generator for polygonal elements written in matlab, Structural and Multidisciplinary Optimization 45 (3) (2012) 309-328. doi:10.1007/s00158-011-0706-z 